\title{
Bloodstream Bacterial Infection in Neutropenic Acute Leukemia Patients
}

\author{
Salwa I. Abedelnasser ${ }^{1 *}$, Hala F. Mohamed ${ }^{1,2}$, Asmaa M. Zahran ${ }^{3}$ \\ ${ }^{1}$ Department of Botany and Microbiology, Faculty of Science (Girls Branch), University of Al-Azhar, Cairo, Egypt \\ ${ }^{2}$ Third Institute of Oceanography, Natural Resources, Xiamen, China \\ ${ }^{3}$ South Egypt Cancer Institute, Assiut University, Assuit, Egypt \\ Email: ^salwaibrahimsalwadream84@yahoo.com
}

How to cite this paper: Abedelnasser, S.I., Mohamed, H.F. and Zahran, A.M. (2020) Bloodstream Bacterial Infection in Neutropenic Acute Leukemia Patients. Journal of Cancer Therapy, 11, 296-305. https://doi.org/10.4236/jct.2020.115024

Received: April 17, 2020

Accepted: May 10, 2020

Published: May 13, 2020

Copyright (c) 2020 by author(s) and Scientific Research Publishing Inc. This work is licensed under the Creative Commons Attribution International License (CC BY 4.0).

http://creativecommons.org/licenses/by/4.0/

\section{(cc) (i) Open Access}

\begin{abstract}
Objective: The rapid detection of blood stream infection has the potential to not only improve patient outcomes due to quicker administration of appropriate antibiotics but also improve antibiotic stewardship by reducing patient exposures to ineffective or unnecessary broad-spectrum antibiotics. Methods: In this study blood samples from acute leukemia neutropenic patients (samples under study) and non neutropenic patients (control) were tested using cultural and non-cultural based techniques via blood culture, C-reactive protein (CRP), Erythrocyte sedimentation rate (ESR) and Molecular techniques (bacterial 16S rDNA analysis). Results: About 22\% \& 15\% were shown positive in blood culturing; 94\% \& $85 \%$ were positive for CRP; $86 \%$ and $75 \%$ were presented by elevation in ESR rate for the first hour; $94 \%$ and $80 \%$ were presented by elevation in ESR rate for second hour. Treatment response of the positive cases by blood culture only was found to be $72.7 \%$ and $100 \%$ not in remission for samples under study and control respectively. In cases under study there is statistically significant correlation between culture growth sensitivity and PCR technique with $\mathrm{P}$ value $<0.005$.
\end{abstract}

\section{Keywords}

Bloodstream Infection (BSI), Blood Cultures, Biomarkers, Molecular Techniques

\section{Introduction}

Bloodstream infection (BSI) in neutropenic patients, is a severe complication and is associated with increased mortality. BSI is routinely diagnosed with blood culture, which detects only culturable pathogens [1]. BSI is treated with broad-spectrum of empirical antimicrobials, due to the lack of specificity or resistance mechanisms 
systemic infections. The antimicrobials are often not efficient against the invading microbes [2] [3] and [4], and its treatment might be inappropriate due to the lack of coverage of the underlying pathogen(s), or the causative pathogens antimicrobial resistance [5]. Although Blood culture only detects culturable pathogens and represents a narrow spectrum of the microbes present in a sample, it requires relatively large volumes of samples [6]. About $75 \%$ from febrile neutropenia, and $50 \%$ of blood cultures from septic shock are positive [1] [7] and [8]. In hemato-logical malignancies the most commonly detected pathogens are bacteria, also fungal and viral infections represent in major complications [1] [6] and [9].

In patients who have bloodstream infections, the viable microorganisms are present in blood [10]. In peripheral blood the density of microorganisms in adults may be as low as ten microorganisms per milliliter and about $100 \mathrm{mi}$ croorganisms per milliliter in children [11]. In principle, the sensitivity of blood cultures is enough to detect these low amounts of microorganisms; however, the density varies during the course of disease, and therefore the blood culture diagnostics will not always yield positive results; therefore recommendation for culturing sufficient quantities of blood is in patients with suspected sepsis [12].

Blood cultures have several advantages. First, they have been in use for more than 100 years and are well integrated in the clinical workflow and clinical guidelines. Second, semi-automated culture systems have greatly simplified handling in the microbiological laboratory which results in a short hands-on time. Third, a wide range of bacterial and fungal pathogens can be isolated and identified [13]. Furthermore, isolation of the pathogen is a prerequisite to phenotypic susceptibility testing which enables clinicians to initiate targeted antimicrobial therapy. However, there are limitations in blood culture diagnostics: detection is limited to pathogens that have the ability to grow in blood cultures. Some microorganisms, such as Legionella spp., Bartonella spp., and Aspergillus spp., grow poorly in blood culture medium. Furthermore, antimicrobials may cause growth inhibition and relevant pathogens may thus go undetected, after the initiation of antimicrobial therapy [14] [15]. Most importantly, many clinicians feel that results are available too late to guide therapy because of blood culture diagnostics requires some time until results are available [16].

Biomarkers, such as C-reactive protein or procalcitonin, aid in the diagnosis of sepsis and they are usually available before microbiological test results. Unfortunately, currently available biomarkers have a low sensitivity and specificity [17] [18] and [19].

Molecular techniques have been developed with the aim to carryout sensitivity progression and to detect bloodstream infection earlier [20] [21].

In pneumococcal pneumonia, blood cultures often remain negative and polymerase chain reaction (PCR)-based detection of Streptococcus pneumoniae in patients with a clinical suspicion for pneumonia has been shown to be more sensitive than blood cultures in clinical studies [22] [23]. 


\section{Patients and Methods}

Fifty new cases of Acute Leukemia Patients with Febrile Neutropenia and twenty patients with acute leukemia without neutropenia of the age, sex and diagnosis (as control), who were admitted to Medical Oncology and pediatric Departments at South Egypt Cancer Institute (SECI) Assiut University (from May 2017 to May 2019).

\subsection{The Clinical Data at Presentation}

Clinical data including age, sex, and presenting clinical features (lymph node enlargement, Splenomegaly, fever and infection, renal problems, liver problems, and Anemia).

\subsection{Material and Methods}

Blood of $(10$ - 15) $\mathrm{ml}$ was collected by either a venous or arterial draw at the same time as the routine clinical blood samples using the same needle stick.

\subsubsection{Complete Blood Count}

Small aliquots $2 \mathrm{ml}$ blood was inoculated to K-EDTA, and performed by using CELL-DYN ${ }^{\circledR}$ CD-3500 CS. ABBOTT DIAGNOSTICS. USA, PENTRA DF NEXUS. SN (60SPN0694) HORIBA \& manual differential count.

\subsubsection{Culture Growth and Sensitivity}

Blood of $3-5 \mathrm{ml}$ inoculated in to blood culture media, Small aliquots was taken for culturing on solid media and for gram stain, identification and determination of sensitivity to antibiotic were performed by using VITEK $^{\circledR} 2$ System (BIOMERIEUX) SN (510774-3EN1) USA.

\subsubsection{Creactive Protein (CRP)}

Serum samples were taken and performed by bioscien CRP latex kit Qualitative and Semi-Qualitative test.

\subsubsection{Erythrocyte Sedimentation Rate (ESR)}

Blood of $1.6 \mathrm{ml}$ was inoculated in Tube containing $0.4 \mathrm{ml}$ of sodium citrate solution. ESR is carried out by using LENA S.N (002164) Barcelona Spain.

\subsubsection{Identification of Bacteria Up to Species Level Using $16 \mathrm{~S}$ rRNA Analysis}

Molecular techniques including DNA extraction PCR mediated amplification of $16 \mathrm{~S}$ ribosomal DNA, purification of PCR products and sequencing of the PCR products for the isolated Bactria under study were performed.

DNA extraction was performed using Gene Jet genomic DNA purification Kit (Thermo K27300). The 16S rRNA amplified by polymerase chain reaction (PCR) using eubacterial universal primers F: 5'-GGT TAC CTT GTT ACG ACT 3'.

Then PCR was performed using MyTaq PCR Red Mix (thermo K27300), Puri- 
fication of PCR product using Gene JET ${ }^{\text {me }}$ PCR Purification Kit (Thermo K0701) and The sequencing of the PCR product was mad in GATC German Company using ABI $3730 \mathrm{xl}$ DNA sequencer by forward and reverse primers, F: 5'-AGA GTT TGA TCC TGG CTC AG-3' and R: 5'-GGT TAC CTT GTT ACG ACTT 3 .

\section{Results and Discussion}

Seventy new cases of leukemia patients (AL) haven't received (chemo or radio) therapy presented by fever and infection, 50 cases of neutropenic acute leukemia patients with the mean age $(13.26 \pm 11.67)$ years and age range from 1 to 46 years old, 37 (74\%) patients were younger than 18 years old, are compared to 20 cases of non neutropenic acute leukemia patients with mean age (20.55 \pm 19.061$)$ years and age range from 3 to 64 years old, 13 (65\%) patients were younger than 18 years old.

Among neutropenic acute leukemia patients, 29 patients (58\%) were males and 21 patients $(42 \%)$ were females, $33(66 \%)$ patients were diagnosed as acute lymphoblastic leukemia (ALL) and 17 (34\%) were diagnosed as acute myeloid leukemia (AML), 47patients (94\%) positive for CRP test, 43 patients (86\%) and 47 patients (94\%) presented by elevation in ESR rate for first and second hour respectively.

Among non neutropenic acute leukemia patients, 12 patients (60\%) were males and 8 patients (40\%) were females, $9(45 \%)$ patients were diagnosed as (ALL) and 11 (55\%) were diagnosed as (AML), 17 patients (85\%) positive for CRP test, 15 patients $(75 \%)$ and 16 patients (80\%) presented by elevation in ESR rate for first and second hour respectively (Table $1 \&$ Table 2 ).

The overall proportion of infected cases positive by blood culture was found

Table 1. The distribution of demographic data of acute leukemia patients.

\begin{tabular}{ccccc}
\hline \multirow{2}{*}{ Variable } & \multicolumn{2}{c}{ ALFNP Cases $(\mathrm{n}=50)$} & \multicolumn{2}{c}{ ALNNP Cases $(\mathrm{n}=\mathbf{2 0})$} \\
\cline { 2 - 5 } & No & $\%$ & No & $\%$ \\
\hline Sex & 29 & 58 & 12 & 60 \\
Male & 21 & 42 & 8 & 40 \\
Female & & & & \\
Age/years & $13.26 \pm 11.67$ & & $20.55 \pm 19.061$ & \\
Mean \pm SD & $1-46$ & & $3-64$ & \\
Range & & & & 45 \\
Diagnosis & 33 & 66 & 9 & 55 \\
ALL & 17 & 34 & 11 & \\
AML & &
\end{tabular}

Abbreviations: (ALFNP) acute leukemia patients with febrile neutropenia \& (ALNNP) acute leukemia patients without neutropenia. 
to be $(22 \%)$ for neutropenic acute leukemia patients, and (15\%) for non neutropenic acute leukemia patients. Among gram positive bacteria (GPB) are, methicillin resistant Staphylococcus aureus, Staphylococcus warneri, Staphylococcus lentus, Staphylococcus lugdunensis, Staphylococcus xylosus, Staphylococcus pseudintermedius, Staphylococcus homini \& Lactococcus garvieae. Among gram negative bacteria (GNB) are, non-fermenting gram-negative Sphingomonas paucimobilis, Klebsiella pneumonia, Enterobacter aerogenes \& Escherichia coli (Table 3 \& Table 4).

The overall proportion of patients after treatment: for neutropenic acute leukemia patients, 27 patients (54\%) were in remission while 23 patients (46\%) were not. For non neutropenic acute leukemia patients, 11 patients (55\%) were in remission while 9 patients (45\%) were not.

Concerning to age, there is no significant correlation between neutropenic

Table 2. The distribution of acute leukemia patients concerning to laboratory investigation.

\begin{tabular}{ccccc}
\hline \multirow{2}{*}{ Laboratory investigations } & \multicolumn{2}{c}{ ALFNP } & \multicolumn{2}{c}{ ALNNP } \\
\cline { 2 - 5 } & Mean \pm SD & Range & Mean \pm SD & Range \\
\hline ESR 1 $^{\text {sthr }}(\mathrm{mm})$ & $54.23 \pm 39.107$ & $3-129$ & $39.65 \pm 41.675$ & $3-115$ \\
$\operatorname{ESR~2}^{\text {ndhr }}(\mathrm{mm})$ & $59.88 \pm 52.756$ & $7-150$ & $62.55 \pm 51.806$ & $7-150$ \\
CRP $(\mathrm{mg} / \mathrm{dl})$ & $60.37 \pm 36.304$ & $3-96$ & $35.1 \pm 30.487$ & $6-96$ \\
\hline
\end{tabular}

Table 3. The distribution of isolated bacteria in acute leukemia febrile neutropenia cases, according to culture growth.

\begin{tabular}{ccc}
\hline Acute leukemia with neutropenia (patients) isolated bacteria & No. & $\%$ \\
\hline Lactococcus garvieae & 1 & 8.3 \\
Staphylococcus warneri & 1 & 8.3 \\
Staphylococcus aureus & 2 & 16.7 \\
Staphylococcus hominis & 2 & 16.7 \\
Staphylococcus pseudintermedius & 1 & 8.3 \\
Staphylococcus lentus & 1 & 8.3 \\
Sphingomonas paucimobilis & 1 & 8.3 \\
Escherichia coli & 1 & 8.3 \\
Klebsiella pneumonia & 1 & 8.3 \\
Enterobacter aerogenes & 1 & 8.3 \\
\hline
\end{tabular}

Table 4. The distribution of isolated bacteria in acute leukemia without neutropenia (ALNNP) cases, according to culture growth.

\begin{tabular}{ccc}
\hline Acute leukemia without neutropenia (ALNNP) isolated bacteria & No. & $\%$ \\
\hline Staphylococcus lugdunensis & 1 & 33.3 \\
Staphylococcus xylosus & 1 & 33.3 \\
Staphylococcus lentus & 1 & 33.3 \\
\hline
\end{tabular}


acute leukemia patients and non neutropenic acute leukemia patients with a $\mathrm{P}$ value of $0.303 \& 0.211$ respectively.

Concerning to sex, there are statistically significance in neutropenic acute leukemia patients and no significant correlation in non neutropenic acute leukemia patients with a $\mathrm{P}$ value $0.003 \& 0.565$ respectively.

According to type of AL, was found to be 4 patients (33.3) ALL \& 8 patients (66.7) AML for patients group, and 0 patients (0) ALL \& 3 patients (100) AML for non neutropenic acute leukemia patients. i.e. percentage of ALL:AML (1:2) for neutropenic acute leukemia patients \& (0:1) AML for non neutropenic acute leukemia patients.

Concerning to type of AL there are statistically significant in the two groups with a $P$ value $0.004 \& 0.03$ respectively.

Concerning to the type of isolated bacteria in neutropenic acute leukemia patients, we found that Gram-positive bacteria (GPB) was the most abundant microorganisms than gram negative bacteria (GNB); it represents about 2:1 while in non neutropenic acute leukemia patients growth only GPB were present (Figure 1 \& Figure 2).

Concerning to the inflammatory biomarkers such as CRP, there is no significant correlation between neutropenic acute leukemia patients and non neutropenic acute leukemia patients with a $\mathrm{P}$ value of $0.480 \& 0.222$ respectively.

Concerning to the ESR ( $1^{\text {st }} \& 2^{\text {nd }}$ hour), there is no significant correlation in neutropenic acute leukemia patients with a $\mathrm{P}$ value of $0.071 \& 0.084$ respectively but there is statistically significant correlation in the non neutropenic acute leukemia patients with a $\mathrm{P}$ value of 0.018 \& 0.034 respectively.

Concerning to treatment response of the cases shown positive by blood culture, only (27.3\%) are found to be in remission and (72.7\%) are not in remission, while all patients $(100 \%)$ for non neutropenic acute leukemia patients are not in remission (Figure $3 \&$ Figure 4 ).

There is no significant correlation in neutropenic acute leukemia patients with a $P$ value of 0.330 , but there are statistically significant in the non neutropenic acute leukemia patients with a $\mathrm{P}$ value of 0.039 .

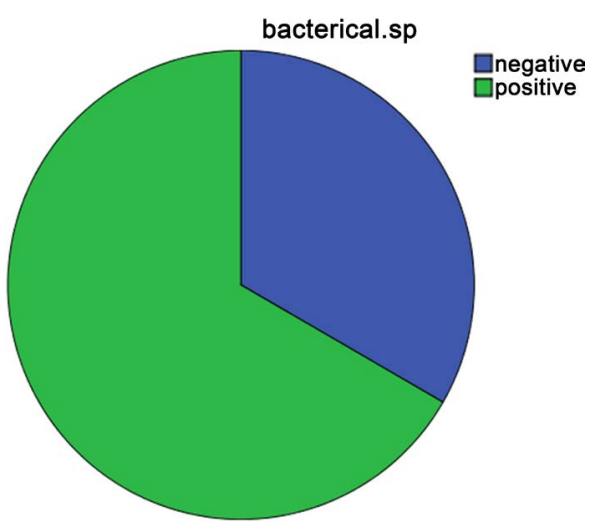

Figure 1. The distribution of isolated bacteria in acute leukemia febrile neutropenia cases, according culture growth. 


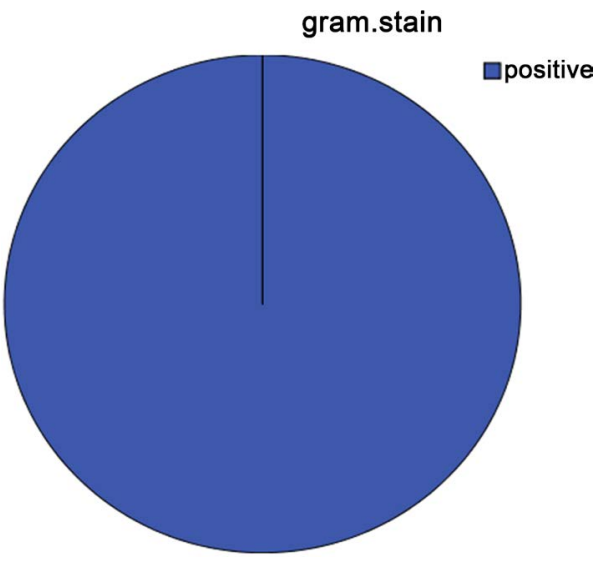

Figure 2. The Distribution of isolated bacteria in acute leukemia non neutropenic cases, according culture growth.

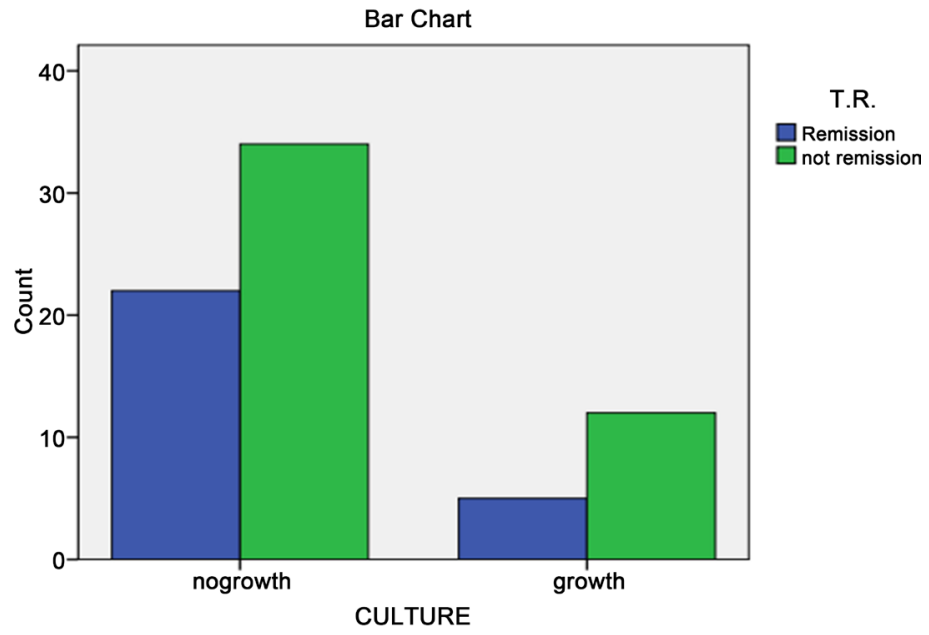

Figure 3. Correlation between culture growth sensitivity and treatment response in acute leukemia febrile neutropenia patients.

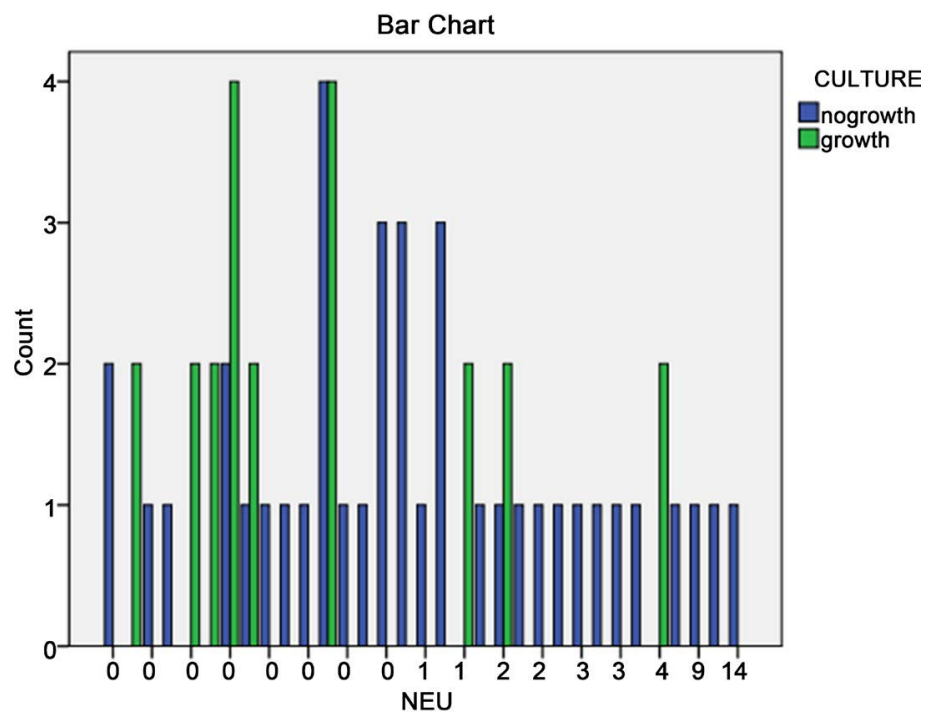

Figure 4. Correlation between culture growth sensitivity and neutrophils in acute leukemia febrile neutropenia patients. 


\section{Molecular technique}

Correlation between culture growth sensitivity and Molecular techniques

Concerning to identification of blood stream bacterial species using PCR technique, we found that there is statistically significant correlation between culture growth sensitivity and PCR with $\mathrm{P}$ value $<0.005$, this is mainly due to about (96\%) opositive samples by blood culture were also positive by PCR, indeed 2 cases out of 50 were positive by PCR but negative by blood culture.

The overall of isolated bacteria from neutropenic acute leukemia patients according to PCR test were staphylococcus aureus, Sphingomonas paucimobilis, Escherichia coli and klebsiella pneumonia.

The infection in neutropenic acute leukemia patients is more incident and associated with more virulence and resistance bacteria than non neutropenic acute leukemia patients.

Not surprisingly, this fundamental principle was associated with high mortality rate among all patients, because that administration of inadequate or inappropriate antimicrobial treatment was also associated with increased hospital mortality.

In the agreement of our study [24] reported that resistance to common antimicrobial agents is being encountered increasingly at most hospitals, in part because of heavy use of antibiotics.

Similarly [25] reported that prophylactic antibiotics have demonstrated some efficacy in reducing the risk of febrile episodes in neutropenic patients with cancer; however, these agents have been associated with additional toxicity and the emergence of antibiotic-resistant bacteria.

\section{Conclusions}

The infection in neutropenic acute leukemia patients is more prevalent and associated with more virulence and resistance of gram positive bacteria than non neutropenic acute leukemia patients. The bacterial species associated with neutropenic acute leukemia patients are more resistant to antibiotic than those associated with non neutropenic acute leukemia patients.

Concerning treatment response of the cases positive for bacterial growth, the remission rate was less in neutropenic acute leukemia than non neutropenic acute leukemia patients. So the earlier detection of the infection the faster prognosis of the disease.

\section{Conflicts of Interest}

The authors declare no conflicts of interest regarding the publication of this paper.

\section{References}

[1] Potgieter, M., Bester, J., Kell, D.B. and Pretorius, E. (2015) The Dormant Blood Microbiome in Chronic, Inflammatory Diseases. FEMS Microbiology Reviews, 39, 
567-591. https://doi.org/10.1093/femsre/fuv013

[2] De Naurois, J., Novitzky-Basso, I., Gill, M.J., Marti, F.M., Cullen, M.H., Roila, F. and ESMO Guidelines Working Group (2010) Management of Febrile Neutropenia: ESMO Clinical Practice Guidelines. Annals of Oncology, 21, v252-v256. https://doi.org/10.1093/annonc/mdq196

[3] Moghnieh, R., Kanafani, Z.A., Abi Hanna, P., Matar, M.J., Mokhbat, J., Jradeh, M., Choucair, J., et al. (2017) 2016 Lebanese Society of Infectious Diseases and Clinical Microbiology Guidelines on the Management of Febrile Neutropenia in Adult Cancer Patients in the Era of Growing Antimicrobial Resistance. Lebanese Medical Journal, 103, 1-20. https://doi.org/10.12816/0038540

[4] Anderson, K., Bradford, N., Edwards, R., Nicholson, J., Lockwood, L. and Clark, J. (2019) Addressing the Barriers to Optimal Management of Febrile Neutropenia in Children with Cancer. European Journal of Oncology Nursing, 45, Article ID: 101719. https://doi.org/10.1016/j.ejon.2019.101719

[5] Kalich, B.A., Maguire, J.M., Campbell-Bright, S.L., Mehrotra, A., Caffey, T., Tulu, Z., Carson, S.S., et al. (2016) Impact of an Antibiotic-Specific Sepsis Bundle on Appropriate and Timely Antibiotic Administration for Severe Sepsis in the Emergency Department. The Journal of Emergency Medicine, 50, 79-88.

https://doi.org/10.1016/j.jemermed.2015.09.007

[6] Gyarmati, P., Kjellander, C., Aust, C., Song, Y., Öhrmalm, L. and Giske, C.G. (2016) Metagenomic Analysis of Bloodstream Infections in Patients with Acute Leukemia and Therapy-Induced Neutropenia. Scientific Reports, 6, Article No. 23532. https://doi.org/10.1038/srep23532

[7] Liese, J.G., Schoen, C., van der Linden, M., Lehmann, L., Goettler, D., Keller, S., Streng, A., et al. (2019) Changes in the Incidence and Bacterial Aetiology of Paediatric Parapneumonic Pleural Effusions/Empyema in Germany, 2010-2017: A Nationwide Surveillance Study. Clinical Microbiology and Infection, 25, 857-864. https://doi.org/10.1016/j.cmi.2018.10.020

[8] Rönkkö, R., Juutilainen, A., Koivula, I., Vänskä, M., Nousiainen, T., Jantunen, E. and Hämäläinen, S. (2018) Changes in the Microbiological Epidemiology of Febrile Neutropenia in Autologous Stem Cell Transplant Recipients. Infectious Diseases, 50, 436-442. https://doi.org/10.1080/23744235.2017.1420221

[9] Öhrmalm, L., Wong, M., Aust, C., Ljungman, P., Norbeck, O., Broliden, K. and Tolfvenstam, T. (2012) Viral Findings in Adult Hematological Patients with Neutropenia. PLoS ONE, 7, e36543. https://doi.org/10.1371/journal.pone.0036543

[10] Chalmers, S.J. and Wylam, M.E. (2020) Methicillin-Resistant Staphylococcus aureus Infection and Treatment Options. In: Methicillin-Resistant Staphylococcus aureus (MRSA) Protocols, Humana, New York, 229-251. https://doi.org/10.1007/978-1-4939-9849-4_16

[11] Bard, J.D. and TeKippe, E.M. (2016) Diagnosis of Bloodstream Infections in Children. Journal of Clinical Microbiology, 54, 1418-1424.

https://doi.org/10.1128/JCM.02919-15

[12] Dellinger, R.P., Levy, M.M., Rhodes, A., Annane, D., Gerlach, H., Opal, S.M., Osborn, T.M., et al. (2013) Surviving Sepsis Campaign: International Guidelines for Management of Severe Sepsis and Septic Shock, 2012. Intensive Care Medicine, 39, 165-228. https://doi.org/10.1007/s00134-012-2769-8

[13] Jordana-Lluch, E., Rivaya, B., Marcó, C., Giménez, M., Quesada, M.D., Escobedo, A., Ausina, V., et al. (2017) Molecular Diagnosis of Bloodstream Infections in Onco-Haematology Patients with PCR/ESI-MS Technology. Journal of Infection, 74, 
187-194. https://doi.org/10.1016/j.jinf.2016.11.011

[14] Liesenfeld, O., Lehman, L., Hunfeld, K.P. and Kost, G. (2014) Molecular Diagnosis of Sepsis: New Aspects and Recent Developments.

[15] Firoozeh, F., Shiralinezhad, A., Momen-Heravi, M., Aghadavod, E. and Zibaei, M. (2019) Rapid Detection of Pathogenic Bacteria in Whole Blood Samples Using 23S rRNA PCR Assays. The Open Microbiology Journal, 13, 101-105. https://doi.org/10.2174/1874285801913010101

[16] Murphy, L., Davidson, S., Chase, J.G., Knopp, J.L., Zhou, T. and Desaive, T. (2020) Patient-Specific Monitoring and Trend Analysis of Model-Based Markers of Fluid Responsiveness in Sepsis: A Proof-of-Concept Animal Study. Annals of Biomedical Engineering, 48, 682-694. https://doi.org/10.1007/s10439-019-02389-9

[17] Lacoma, A., Rodríguez, N., Prat, C., Ruiz-Manzano, J., Andreo, F., Ramírez, A., Domínguez, J., et al. (2012) Usefulness of Consecutive Biomarkers Measurement in the Management of Community-Acquired Pneumonia. European Journal of Clinical Microbiology \& Infectious Diseases, 31, 825-833. https://doi.org/10.1007/s10096-011-1381-0

[18] Marshall, A.C., Castellanos, T.G., Motas, I.M., Rodriguez, D.S., Romaní, M.E.T. and Ávila, J.P. (2014) Utility of C-Reactive Protein and Procalcitonin for Detecting Bloodstream Infection in Patients with HIV/AIDS. World Journal of AIDS, 4, 287. https://doi.org/10.4236/wja.2014.43033

[19] Mori, Y., Miyawaki, K., Kato, K., Takenaka, K., Iwasaki, H., Harada, N., Teshima, T., et al. (2011) Diagnostic Value of Serum Procalcitonin and C-Reactive Protein for Infections after Allogeneic Hematopoietic Stem Cell Transplantation versus Nontransplant Setting. Internal Medicine, 50, 2149-2155.

https://doi.org/10.2169/internalmedicine.50.5798

[20] Mancini, N., Carletti, S., Ghidoli, N., Cichero, P., Burioni, R. and Clementi, M. (2010) The Era of Molecular and Other Non-Culture-Based Methods in Diagnosis of Sepsis. Clinical Microbiology Reviews, 23, 235-251. https://doi.org/10.1128/CMR.00043-09

[21] Paolucci, M., Landini, M.P. and Sambri, V. (2010) Conventional and Molecular Techniques for the Early Diagnosis of Bacteraemia. International Journal of Antimicrobial Agents, 36, S6-S16. https://doi.org/10.1016/j.ijantimicag.2010.11.010

[22] Avni, T., Mansur, N., Leibovici, L. and Paul, M. (2010) PCR Using Blood for Diagnosis of Invasive Pneumococcal Disease: Systematic Review and Meta-Analysis. Journal of Clinical Microbiology, 48, 489-496. https://doi.org/10.1128/JCM.01636-09

[23] Book, M., Lehmann, L.E., Zhang, X. and Stüber, F. (2013) Monitoring Infection: From Blood Culture to Polymerase Chain Reaction (PCR) Best Practice \& Research. Clinical Anaesthesiology, 27, 279-288. https://doi.org/10.1016/j.bpa.2013.06.010

[24] Chastain, D.B., Wheeler, S., Franco-Paredes, C., Olubajo, B. and Hawkins, W.A. (2018) Evaluating Guideline Adherence Regarding Empirical Vancomycin Use in Patients with Neutropenic Fever. International Journal of Infectious Diseases, 69, 88-93. https://doi.org/10.1016/j.ijid.2018.02.016

[25] Almyroudis, N.G., Osawa, R., Samonis, G., Wetzler, M., Wang, E.S., McCarthy, P.L. and Segal, B.H. (2016) Discontinuation of Systematic Surveillance and Contact Precautions for Vancomycin-Resistant Enterococcus (VRE) and Its Impact on the Incidence of VRE Faecium Bacteremia in Patients with Hematologic Malignancies. Infection Control \& Hospital Epidemiology, 37, 398-403. https://doi.org/10.1017/ice.2015.310 